\title{
Smoking, drinking, and incident cognitive impairment: a cohort community based study included in the Gospel Oak project
}

\author{
J A Cervilla, Martin Prince, Anthony Mann
}

\begin{abstract}
Objectives-Recent longitudinal studies have reported that smoking increases risk for cognitive impairment and that moderate alcohol intake could be preventive. The association between both cigarette smoking and alcohol drinking and incident cognitive impairment was studied in a representative population.

Methods-This is a 1 year prospective population based cohort sudy of all residents aged 65 or over in the electoral ward of Gospel Oak in London, UK $(n=889)$. Cognitive impairment was assessed at baseline and 1 year later using the organic brain syndrome (OBS) cognitive impairment scale from the short CARE structured assessment. Subjects who were cognitively impaired at baseline were excluded from this analysis.
\end{abstract}

Results-The prevalence of OBS cognitive impairment was $10.4 \%$ at index assessment and the 1 year cumulative incidence of cognitive impairment was $5.7 \%$. Cognitive impairment was not associated with use of alcohol, although there was a non-significant association in the direction of a protective effect against onset of cognitive impairment for moderate drinkers compared with non-drinkers and heavy drinkers. Current smoking status predicted cognitive impairment (risk ratio (RR) 3.7; (95\% confidence interval $(95 \% \mathrm{CI})=1.1-12.3)$ independently from sex, age, alcohol, occupational class, education, handicap, depression, and baseline cognitive function.

Conclusions-Smoking seems to be a prospective risk factor for incident cognitive impairment; thus encouragement of older people to stop smoking could be considered as part of a strategy to reduce the incidence of cognitive impairment. (F Neurol Neurosurg Psychiatry 2000;68:622-626)

Keywords: smoking; alcohol; dementia; cognitive impairment

Cognitive impairment is the core clinical feature contributing to the diagnosis of dementia. There are strong associations between cognitive impairment and dependency in activities of daily living, ${ }^{1}$ morbidity, ${ }^{2}$ and mortality ${ }^{3}$ in late life. The international classification of diseases, 10th edition (ICD-10) recently recognised mild cognitive impairment as a clinical entity on its own right. ${ }^{4}$ The earlier recognition of the onset of dementia through ascertainment of mild cognitive decline may also offer possibilities for early treatment and delay of disability. ${ }^{5}$

The relation between smoking and cognitive impairment still needs clarification. Several case-control studies initially reported inverse associations between cigarette smoking and the prevalence of Alzheimer's disease. ${ }^{6-8}$ Some of these studies were included in a meta-analysis by the EURODEM group, ${ }^{9}$ which reached the same conclusion (odds ratio (OR) $0.8 ; 95 \%$ confidence interval $(95 \% \mathrm{CI})=0.6-1 \cdot 0)$. A protective effect of smoking was biologically plausible as it had been suggested that enhancement of nicotine neurotransmission might improve performance on selected cognitive tests. ${ }^{10}$ However, selection bias was likely to have affected the findings of these studies. Cerebrovascular symptomatology excluded the diagnosis of probable Alzheimer's disease, but similar exclusion criteria were generally not applied to the control groups. ${ }^{11}{ }^{12}$ Also, there have been failures to confirm the protective effect of smoking in other case-control studies assessing the association with Alzheimer's disease $\mathrm{e}^{13}$ and in cross sectional studies estimating associations with cognitive impairment. ${ }^{15-17}$ Longitudinal studies, including recent findings from the cohort studies completed by the EURODEM consortium, in fact suggest that smoking is a risk factor for Alzheimer's disease ${ }^{18-20}$ and cognitive decline. ${ }^{21-24}$

Similarly, the nature of the relation between alcohol use and cognitive decline is yet to be clarified. Some prospective epidemiological studies have reported that moderate alcohol intake may protect against cognitive decline, ${ }^{212524}$ others that it has no effect on cognitive decline ${ }^{91726}$ or dementia, ${ }^{27}$ or even may be a risk factor for cognitive dysfunction. ${ }^{28}$

In this longitudinal community based study we explored the relation between smoking, alcohol intake, and incident cognitive impairment using a representative sample of elderly subjects for whom prospective data on smoking behaviour and alcohol consumption were gathered before the onset of cognitive impairment. These two risk exposures tend to be associated with each other and are, therefore, assessed as potential confounding factors along with other factors known or suspected to be associated with both exposure and outcome, such as age, sex, education, occupational class, depression, and general physical health status. 
Table 1 Items included in the organic brain syndrome scale $(O B S)$

(1) Doesn't know his age

(2) Doesn't know the year of his birth

(3) Doesn't know the number of years in neighbourhood

(4) Doesn't know his address

(5) Doesn't know the rater's name, first try

(6) Can't recall the President's/Prime Minister's name, current or previous

(7) Doesn't know the month

(8) Doesn't know the year

(9) Doesn't know the rater's name, second try

(10) Failed knee-hand-ear test

\section{Method}

DESIGN

The study design has been reported in detail elsewhere. ${ }^{29}$ In brief, this is a longitudinal prospective study of the entire population aged 65 years or older living in a defined geographic area: the Gospel Oak electoral district of North London, UK. A population register of 889 elderly residents was established by knocking on the doors of all eligible households in 1993. Interviews were conducted over 8 months in 1994, and follow up interviews over a similar period 1 year later.

\section{EXPOSURES}

Smoking and drinking

Subjects were asked whether they had ever smoked and one of three answers was recorded: never smoked, smoked in the past, or current smoker. Among those who smoked, currently or in the past, information was obtained on the average number of cigarettes smoked a day and on the number of years they had smoked for. Participants were also asked what was the most alcohol they would drink in an average week before the age of 65, after the age of 65 , and during the week before the first interview. The amount of alcohol use was recorded in units (1 unit is equivalent to a glass of wine or a single measure of spirits).

POTENTIAL CONFOUNDERS

At baseline (1994) subjects were interviewed using the short CARE package, which contains several clinical indicator scales including the organic brain syndrome scale (OBS) assessing cognitive impairment (see later), the activity limitation scale, and the depression diagnostic scale (DPDS). ${ }^{30}$ As described elsewhere, ${ }^{31} 17 \%$ of subjects scoring 7 or more points in the DPDS were considered to have pervasive depression. The level of handicap was assessed using the London handicap scale. ${ }^{32}$ Information on sex, age, years of formal education (less than 9 years $v 9$ to 11 years $v$ more than 11 years) and occupational class (managerial/ professional, skilled not manual, skilled manual, unskilled) was also gathered at baseline.

\section{INCIDENT COGNITIVE IMPAIRMENT (OUTCOME} VARIABLE)

Cognitive impairment was measured, both at time 1 (1994) and at time 2 (1995), using the OBS. ${ }^{30}$ The OBS has been reported to be a valid and reliable scale in the assessment of cognitive impairment in elderly community samples. ${ }^{30} 33{ }^{34}$ Table 1 shows all OBS items. In determining cognitive impairment we used the suggested cut off of 4 or more points on the OBS that has shown satisfactory criterion validity against a clinical assessment, via an informant interview, of the presence of clinically significant cognitive impairment. This cut off point produces a negative predictive value of $87 \%$, a positive predictive value of $30 \%$, a specificity of $90 \%$, and a sensitivity of $25 \% .^{33}$ Construct and concurrent validity has also been reported with high correlations between the OBS and global rating of cognitive impairment by clinicians (coefficient $=0.71$ ) and between the OBS and a diagnosis established by a trained gerontologist after interviewing an informant (0.69). ${ }^{33}$ The OBS has also been reported as a reliable measure for cognitive impairment demonstrating high internal consistency $(\alpha=0.84)$ and good interrater reliability $(\kappa=0.78) .{ }^{30}{ }^{33}{ }^{34}$ Participants who scored 4 or more points on the OBS at baseline were considered to be cognitively impaired and excluded from this analysis. We then compared the characteristics of those who developed cognitive impairment 1 year later, with those who remained cognitively unimpaired throughout.

STATISTICAL ANALYSIS

We first explored the univariate associations between the OBS cognitive impairment and all

Table 2 Multivariate associations between smoking/drinking and incident cognitive impairment *

\begin{tabular}{|c|c|c|c|c|c|c|c|c|c|}
\hline & \multicolumn{3}{|l|}{ Crude } & \multicolumn{3}{|c|}{ Adjusted model 1} & \multicolumn{3}{|c|}{ Adjusted model 2} \\
\hline & $R R$ & $95 \% C I$ & $p$ Value & $R R$ & $95 \% C I$ & $p$ Value & $R R$ & $95 \% C I$ & $p$ Value \\
\hline Smoking & & & 0.055 & & & 0.047 & & & 0.035 \\
\hline Never smoked & Base & & & Base & & & Base & & \\
\hline Ex-smoker & 0.74 & $0.3-2.1$ & 0.6 & 0.98 & $0.3-3.3$ & 0.96 & 0.86 & $2-2.9$ & 0.81 \\
\hline Current smoker & 2.3 & $0.8-6.3$ & 0.07 & 3.6 & $0.99-13.5$ & 0.048 & 4.07 & $1.01-16.3$ & 0.047 \\
\hline Alcohol before the age of 65 & & & 0.54 & & & 0.58 & & & \\
\hline 0 units & Base & & & Base & & & & & \\
\hline $1-10$ units & 0.75 & $0.3-1.9$ & 0.55 & 0.74 & $0.2-2.1$ & 0.58 & & & \\
\hline $11-30$ units & 0.25 & $0.3-2.0$ & 0.19 & 0.21 & $0.1-1.9$ & 0.17 & & & \\
\hline $30+$ units & 1.16 & $0.3-4.3$ & 0.82 & 0.81 & $0.2-4.2$ & 0.80 & & & \\
\hline Alcohol after the age of 65 & & & 0.55 & & & & & & 0.51 \\
\hline 0 units & Base & & & & & & Base & & \\
\hline $1-10$ units & 0.52 & $0.2-1.4$ & 0.18 & & & & 0.43 & $0.1-1.4$ & 0.16 \\
\hline $11-30$ units & 0.54 & $0.1-2.5$ & 0.43 & & & & 0.51 & $0.1-3.1$ & 0.46 \\
\hline $30+$ units & 0.01 & $0.0-6.2$ & 0.76 & & & & 0.01 & $0.0-2.5$ & 0.68 \\
\hline
\end{tabular}

^Both models (1 and 2) are adjusted for age, sex, baseline OBS score, baseline depression, occupational class, education, and handicap.

Model 1includes smoking and alcohol before age of 65; model 2 includes smoking and alcohol after age of 65 .

$\mathrm{RR}=$ risk ratio; significant $\mathrm{p}$ values are in bold. 
Table 3 Univariate associations with incident cognitive impairment (CI) *

\begin{tabular}{|c|c|c|c|}
\hline Independent variable & $\begin{array}{l}\text { Mean or } n \text { (\% with } \\
\text { CI) }\end{array}$ & $\begin{array}{l}\text { Student's tor } \\
\chi^{2}\end{array}$ & $p$ Value \\
\hline Age & $-4.33(\mathrm{SE} 1.4) \dagger$ & -3.03 & 0.003 \\
\hline Non-CI group & $74.8(\mathrm{SD} 6.7)$ & & \\
\hline CI group & $78.7(\mathrm{SD} 8.1)$ & & \\
\hline Age (decades) & & $13.15 \ddagger$ & 0.0001 \\
\hline 65 to $75 \mathrm{y}$ & $7 / 249(2.8 \%)$ & & \\
\hline 76 to $85 \mathrm{y}$ & $12 / 141(8.5 \%)$ & & \\
\hline 86 to 95 or more $y$ & $5 / 28(17.9 \%)$ & & \\
\hline Sex & & 0.017 & 0.9 \\
\hline Male & $9 / 162(5.6 \%)$ & & \\
\hline Female & $15 / 256(5.9 \%)$ & & \\
\hline Activity limitation scale & & $5.7 \ddagger$ & 0.017 \\
\hline Lower quarter & $4 / 149(2.7 \%)$ & & \\
\hline 2nd quarter & $4 / 83(4.8 \%)$ & & \\
\hline 3rd quarter & $9 / 114(7.9 \%)$ & & \\
\hline Higher quarter & $7 / 72(9.7 \%)$ & & \\
\hline London handicap scale & & $13.6 \ddagger$ & 0.0001 \\
\hline Lower quarter (least handicapped) & $3 / 116(2.6 \%)$ & & \\
\hline 2nd quarter & $3 / 121(2.5 \%)$ & & \\
\hline 3rd quarter & $6 / 113(5.3 \%)$ & & \\
\hline Higher quarter (most handicapped) & $11 / 65(17 \%)$ & & \\
\hline Alcohol use $(<65 \mathrm{y})$ & & 0.02 & 0.89 \\
\hline 0 units & $11 / 164(6.7 \%)$ & & \\
\hline $1-10$ units & $8 / 156(5 \%)$ & & \\
\hline $11-30$ units & $1 / 57(4.3 \%)$ & & \\
\hline $31+$ units & $3 / 39(7.7 \%)$ & & \\
\hline Alcohol use $(>65 \mathrm{y})$ & & 2.77 & 0.42 \\
\hline 0 units & $16 / 213(7.5 \%)$ & & \\
\hline $1-10$ units & $6 / 147(4 \%)$ & & \\
\hline $11-30$ units & $2 / 47(4.3 \%)$ & & \\
\hline $31+$ units & $0 / 10(0 \%)$ & & \\
\hline Smoking & & 5.80 & 0.055 \\
\hline Never & $7 / 134(5.2 \%)$ & & \\
\hline Ex-smoker & $8 / 204(3.9 \%)$ & & \\
\hline Current smoker & $9 / 80(11.25 \%)$ & & \\
\hline DPDS depression at time 1 & & 0.2 & 0.65 \\
\hline Non-depressed & $20 / 361(5.6 \%)$ & & \\
\hline Depressed & $4 / 57(7 \%)$ & & \\
\hline Education (y) & & 0.77 & 0.68 \\
\hline$<9 \mathrm{y}$ & $3 / 35(12.5 \%)$ & & \\
\hline $9-11$ y & $17 / 293(5.8 \%)$ & & \\
\hline$>11$ y $(\mathrm{CI})$ & $4 / 89(4.5 \%)$ & & \\
\hline Social (occupational) class & & $4.13 \ddagger$ & 0.042 \\
\hline Professional/managerial & $3 / 89(3.4 \%)$ & & \\
\hline Skilled non-manual & $4 / 114(3.5 \%)$ & & \\
\hline Skilled manual & $7 / 122(5.7 \%)$ & & \\
\hline Semiskilled/unskilled & $9 / 92(9.8 \%)$ & & \\
\hline
\end{tabular}

$\star_{t}$ Tests or $\chi^{2}$ as appropriate.

†Mean difference (SE); $¥ \chi^{2}$ statistic for linear trend; DPDS=depression scale (short CARE).

exposures and potential confounders using the Student's $t$ test or the $\chi^{2}$ test as appropriate. Relative risk (RR) values and Cornfield 95\% CIs were then calculated using the traditional method of 2 by $\mathrm{K}$ tables analysis. We decided a priori to control for potential confounders using logistic regression, building two models, one including smoking and alcohol before the age of 65 and the other including smoking and alcohol after the age of 65 . Both models were adjusted for age, sex, time 1 OBS score, time 1 depression, occupational class, education, and handicap. We finally tested whether sex modified the effect of drinking or smoking over incident cognitive impairment.

\section{Results}

RESPONSE AND REPRESENTATIVENESS

At baseline, 654 subjects were interviewed, representing $74 \%$ of all 889 persons who qualified for the study sample; 127 subjects $(14 \%)$ refused an interview, $54(6 \%)$ proved elusive without directly refusing, and $51(6 \%)$ had died after the census date but before interview could be carried out. One year after baseline assessment, 451 (69\% of the 654 baseline participants) were reinterviewed, representing $75 \%$ of those that had survived (598). Out of those that had not been followed up 56 had died $(9 \%), 78(12 \%)$ had refused reinterview, $30(5 \%)$ had moved out of the study area, 16 $(2 \%)$ could not be found, and $22(3 \%)$ could not be reinterviewed. Low income was associated with refusal (OR 3.0; 95\% CI 1.8-5.5), whereas living alone was inversely associated with refusal. Those that were not included in the follow up study did not vary from the follow up group by sex, age, smoking, or drinking status. Low scores on the OBS cognitive impairment scale at baseline predicted death within the year of the study, but not refusal to participate at follow up.

OUTCOME MEASURE: INCIDENT COGNITIVE

IMPAIRMENT

The prevalence of OBS cognitive impairment at onset in this sample was $10.4 \%(68 / 654)$. Out of the 451 subjects that were followed up, $34(7.5 \%)$ were cognitively impaired at onset and were excluded from the analysis. The 1 year cumulative incidence of cognitive impairement at time 2 was $5.7 \%(24 / 417)$.

\section{UNIVARIATE ASSOCIATIONS WITH INCIDENT} COGNITIVE IMPAIRMENT

There was a nearly significant trend for an association between cigarette smoking and incident cognitive impairment $(\mathrm{p}=0.055)$. Thus, current smokers were more likely to be cognitively impaired than never smokers ( $R R$ 2.3; 95\% CI=0.85-6.3; p=0.07), whereas ex-smokers showed no additional risk for incident cognitive impairment when compared with never smokers (RR 0.74; 95\% CI $=0.3-$ $2.1 ; \mathrm{p}=0.6$, table 2). There was a nonsignificant trend for a lower frequency of incident cognitive impairment in those subjects who drank alcohol moderately (1 to 30 units a week) compared with those who never drank or who drank excessively (more than 30 units a week), both before and after the age of 65 (table 2).

Incident cognitive impairment was significantly associated with older age and with functional limitations (activity limitation scale) and handicap (table 3). We also found a linear association between increasingly low social (occupational) class and cognitive impairment $\left(\chi^{2}\right.$ for trend $=4.13$; OR for each decreasing step in social class $=1.54 ; 95 \% \mathrm{CI}=1.01-2.35$; $\mathrm{p}=0.04)$. We found no associations between cognitive impairment and either depression at onset, sex, or educational level (table 3 ).

\section{CONTROLLING FOR CONFOUNDERS}

Table 2 shows the crude and adjusted associations between smoking or drinking and incident cognitive impairment. After adjusting for age, sex, time 1 OBS score, time 1 depression, occupational class, education, handicap, and either alcohol before the age of 65 (model 1) or after the age of 65 (model 2), those subjects who were current smokers were at a significantly higher risk of being cognitively impaired than never smokers or ex-smokers. We did not find significant differences in the dose of smoking between current smokers who had incident cognitive impairment and those current smokers who were not cognitively im- 
paired. Within the same multivariate models, alcohol drinking (before or after the age of 65) was not a risk factor for incident cognitive impairment, although there was a nonsignificant trend for a protective effect. We did not find an interaction by sex in the risk conferred by smoking for cognitive impairment $\left(\chi^{2}\right.$ interaction $\left.=0.05 ; p=0.97\right)$. Social class and education did not confound or modify the effects of either smoking or alcohol consumption on onset of cognitive impairment.

\section{Discussion}

STUDY DESIGN AND RESPONSE

Given the prospective cohort study design, unlike cross sectional or case-control studies our study is less likely to generate information bias and allows assessment of direction of causality. However, some limitations should be acknowledged. ${ }^{29}$ Given that Gospel Oak is a typical inner city area with high levels of socioeconomic deprivation, the generalisability of our results might be limited. The response rate at follow up was only $75 \%$ of all survivors, which might have introduced bias. Refusal was associated with low income. Refusal and mortality rates were each higher among subjects who were depressed at index assessment, ${ }^{29}$ possibly explaining the lack of association between baseline depression and incident cognitive impairment. However, for the index assessment the age, sex, marital status, and living circumstances of participants was representative of the target population. ${ }^{31}$ The prevalence rate of cognitive impairment $(10.4 \%)$ is similar to that previously found among subjects older than 65 in the Gospel Oak area by Livingston et $a^{34}(8 \%)$ using the same cut off point in the OBS. Similarly, we found a 1 year cumulative incidence of OBS cognitive impairment of $5.7 \%$ which compares with that of $6.2 \%$ found by a previous study ${ }^{35}$ also using the OBS 4 point cut off, although their follow up period was much longer (mean interval (SD) 28.8 (4.5) months). We must acknowledge that some possiblitiy of partial random error in the assessment of cognitive impairment remains, as the follow up period was only 1 year and cognitive impairment was only measured twice.

\section{ALCOHOL CONSUMPTION}

Although the univariate analysis indicated that subjects who drank alcohol moderately (between 1 to 30 units a week) before the age of 65 seemed less likely to have incident cognitive impairment at follow up than those who never drank, or who used to drink more heavily (31+units per week), differences were not statistically significant and became less apparent after controlling for potential confounders (table 2, model 1). Therefore, we could not replicate previous reports of a protective effect of alcohol against failing cognition..$^{212536}$ Among subjects who drank after the age of 65 the onset of cognitive impairment was less than among those who did not drink but differences were, again, not significant. No onsets of cognitive impairment occurred among those who drank more than 31 units of alcohol a week, perhaps accounted for by a selective mortality effect among heavy drinkers with respect to propensity for cognitive impairment. Our findings broadly support the view that alcohol consumption is neither a protective factor nor a risk factor for cognitive impairment, ${ }^{91726}$ but could also be consistent with a small protective effect not detected because of limited statistical power in this study.

SMOKING AND COGNITIVE IMPAIRMENT

Current smokers were between 3.6 times (model 1) and four times (model 2) more likely to be cognitively impaired than never smokers after adjusting for baseline cognitive function, depression, occupational class, education, handicap, and either alcohol before the age of 65 (model 1) or after the age of 65 (model 2). This is consistent with the view that smoking, which contributes to vascular disease and atherosclerosis, ${ }^{37}$ could increase the risk for dementia and cognitive impairment. ${ }^{38}{ }^{39}$ Previous cross sectional reports suggesting a protective effect $^{6-8}$ have been criticised for their susceptibility to survival bias, selection bias, and reverse causality. ${ }^{11219}$ Our findings support the notion, suggested by other longitudinal studies, ${ }^{192123}$ that cigarette smoking seems to be a risk factor for cognitive impairment. However, this effect may not be sustained as we have recently found that smoking neither increases nor decreases the risk for long term cognitive decline. ${ }^{36}$ In addition, residual confounding and recall bias cannot be entirely ruled out, although the second should be minimal given that we have adjusted for baseline OBS score.

\section{Conclusion}

We did not find an association between alcohol consumption and onset of cognitive impairment. However, our results indicate that persistent cigarette smoking into late life increases the risk for cognitive impairment. Smoking prevention can be a target for preventive interventions against cognitive impairment.

We thank Dr Gill Livingstone and Dr Boothby for their work on earlier phases of the Gospel Oak Project, Dr Harwood for permission to use the London handicap scale and for for his help mission to use the London handicap scale and for for his help with the study, Professor J Copeland and Dr P Saunders for advice on other measures, and Dr N Graham for her continuing support with all aspects of the project. Finally, our thanks go to all the general practitioners and the older residents of Gospel Oak, without whose cooperation this study would have not been
possible. This study was supported by a Wellcome Trust grant.

1 Gill C, Richardson E, Tinetti M. Evaluating the risk of dependence in activities of daily living among communityliving older adults with mild cognitive impairment. $\mathcal{F}$ Gerontol A Biol Sci Med Sci 1995;50:235-41.

2 Weiler PG, Lubben JE, Chi I. Cognitive impairment and hospital use. Am f Public Health 1991;81:1153-7.

3 Liu IY, Lacroix AZ, White L. Cognitive impairment and mortality: a study of possible confounders. Am F Epidemiol 1990;132:136-43.

4 World Health Organisation. The international classification of diseases, 10 th ed. Chapter V: mental and behavioural disorders. London: Churchill Livingstone, 1994.

5 Kukull WA. Problems in measuring and interpreting cognitive decline [editorial]. F Am Geriatr Soc 1998;46:1578-9.

6 Amaducci LA, Fratiglioni L, Rocca WA, et al. Risk factors Amaducci LA, Fratiglioni L, Rocca WA, et al. Risk factors
for clinically diagnosed Alzheimer's disease: a case-control for clinically diagnosed Alzheimer's disease: a case-control
study of an Italian population. Neurology 1986;36:922-31. 7 Graves AB, White E, Koepsell T, et al. A case-control study Graves AB, White E, Koepsell T, et al. A case-control study
of Alzheimer's disease. Ann Neurol 1990;28:1698-707. 
8 van Duijn CM, Hofman A. Relation between nicotine intake

and Alzheimer's disease. BMF;302:1491-4. traves AB, van Duinn CM, Chandra V, et al. Alcohol and tobacco consumption as risk factors for Alzheimer's disease: a collaborative re-analysis of case-control studies. Int f Epidemiol 1991;20(suppl 2):S48-57.

10 Levin ED. Nicotinic systems and cognitive function. Psychopharmacology (Berl) 1992;108:417-31.

11 Riggs JE. Smoking and Alzheimer's disease: protective effect or differential survival bias? Lancet 1993;342:793-4

12 Prince MJ. Vascular risk factors and atherosclerosis as risk factors for cognitive decline and dementia. 7 Psychosom Res 1995;39:525-30.

13 Shalat AL, Seltzer B, Pidcock C, et al. Risk factors for Alzheimer's disease. Neurology 1987;37:1295-300.

14 Brenner DE, Kukull WA, van Belle G, et al. Relationship between Alzheimer's disease and cigarette smoking in a population-based case-control study. Neurology 1993;43: population-

15 Whittington JE, Huppert, FA. Smoking and cognitive decline. Human Psychopharmacology 1997;12:467-80.

16 Kilander L, Nyman H, Boberg M, et al. Hypertension is related to cognitive impairment: a 20-year follow-up of 999 men. $H$ yp

17 Elwood PC, Gallacher JE, Hopkinson C, et al. Smoking, drinking and other lifestyle factors and cognitive function in men in the Caerphilly cohort. $\mathcal{F}$ Epidemiol Community Health 1999;53:9-14.

18 Hebert LE, Scherr PA, Beckett LA, et al., Age specific incidence of Alzheimer's disease in a community population. FAMA 1995;273:1354-9.

19 Launer LJ, Andersen K, Dewey ME, et al. Rates and risks factors for dementia and Alzheimer's disease: results from the EURODEM pooled analyses. Neurology 1999;52:7884.

20 Merchant C, Tang MX, Albert S, et al. The influence of smoking on the risk of Alzheimer's disease. Neurology 1999;52:1408-12.

21 Launer LJ, Feskens EJM, Kalmijn S, et al. Smoking, drinking, and thinking. The Zutphen elderly study. Am $\mathcal{f}$ Epidemiol 1996;143:219-27.

22 Prince $M$, Lewis G, Bird A, et al. A longitudinal study of factors predicting change in cognitive test scores over time, in an older hypertensive population. Psychol Med 1996;26 $555-68$

23 Galanis DJ, Petrovitch H, Launer LJ, et al. Smoking history in middle age and subsequent cognitive performance in elderly Japanase-American men: the Honolulu-Asia aging study. Am F Epidemiol 1997;145:507-15. 24 Carmelli D, Swan GE, Reed T, et al. The effect of apolipo-
protein E e4 in the relationships of smoking and drinking to cognitive function. Neuroepidemiol 1999;18:125-33.
25 Dufouil C, Ducimetiere P, Alperovitch A. Sex differences in the association between alcohol consumption and cognitive the association between alcohol consumption and

26 Edelstein S, Krits-Silverstein D, Barret-Connor E, et al. Prospective association of smoking and alcohol use with cognitive function in and elderly cohort. Fournal of Women's Health 1998;7:1271-81.

27 Broe GA, Creasey H, Jorm AF, et al. Health habits and risk of cognitive impairment and dementia in old age: a prospective study on the effects of exercise, smoking and alcohol consumption. Australia and New Zealand fournal of Public Health 1998;22:621-3.

28 Teri L, Hughes JP, Larson EB. Cognitive deterioration in Alzheimer's disease: behavioural and health factors. F Gerontol 1990;42:3-10.

29 Prince M, Harwood R, Thomas A, et al. A prospective population-based cohort study of the effects of disablement and social milieu on the onset and maintenance of late-life depression. The Gospel Oak Project VII. Psychol Med 1998;28:337-50.

30 Gurland B, Golden RR, Teresi JA, et al. The short CARE: an efficient instrument for the assessment of depression, an efficient instrument for the assessment of dep

31 Prince MJ, Harwood RH, Blizard RA, et al. Impairment, disability and handicap as risk factors for depression in old age. The Gospel Oak Project V. Psychol Med 1997;27:31121

32 Harwood RH, Ebrahim S. Manual of the London handicap scale. Nottingham: University of Nottingham, 1995.

33 Teresi JA, Golden RR, Gurland BJ, et al. Construct validity of indicator scales developed from the comprehensive assessment and referral evaluation interview schedule. $f$ Gerontol 1984;39:147-57.

34 Livingston G, Sax K, Willison J, et al. The Gospel Oak study stage II: the diagnosis of dementia in the community. Psychol Med 1990;20:881-91.

35 Boothby H, Blizard R, Livingston G, et al. The Gospel Oak study stage III: the incidence of dementia. Psychol Med study stage III:

36 Cervilla JA, Prince M, Joels S, et al. Long term predictors of cognitive outcome in a cohort of elderly hypertensive volunteers. Br f Psychol 2000 (in press)

37 Shinton R, Beevers G. Metanalysis of the relation between cigarette smoking and stroke. BMf 1989;298:789-94.

38 Prince MJ, Cullen MC, Mann AH. Risk factors for Alzheimer's disease and dementia: a case-control study based on the MRC elderly hypertension trial. Neurology 1994;44:97-104.

39 Hofman A, Ott A, Breteler MB, et al. Atherosclerosis, apolipoprotein E, and the prevalence of dementia and Alzheimer's disease in the Rotterdam study. Lancet 1997;349:1514. 